\title{
Correction to: Spatial Dependence of Stably Stratified Nocturnal Boundary-Layer Regimes in Complex Terrain
}

\author{
Carsten Abraham ${ }^{1}$ iD $\cdot$ Adam H. Monahan ${ }^{1}$
}

Published online: 7 February 2021

(c) Springer Nature B.V. 2021

\section{Correction to: Boundary-Layer Meteorology (2020) 177:19-47 https://doi.org/10.1007/s10546-020-00532-x}

In the original publication, the same figure was illustrated twice as figures 10 and 11. Consequently, figure 11 was incorrect. The correct version of Fig. 11 is provided in this correction.

The original article has been corrected.

The original article can be found online at https://doi.org/10.1007/s10546-020-00532-x.

Carsten Abraham

abrahamc@uvic.ca

1 School of Earth and Ocean Sciences, University of Victoria, P.O. Box 3065 STN CSC, Victoria, BC V8P 5C2, Canada 

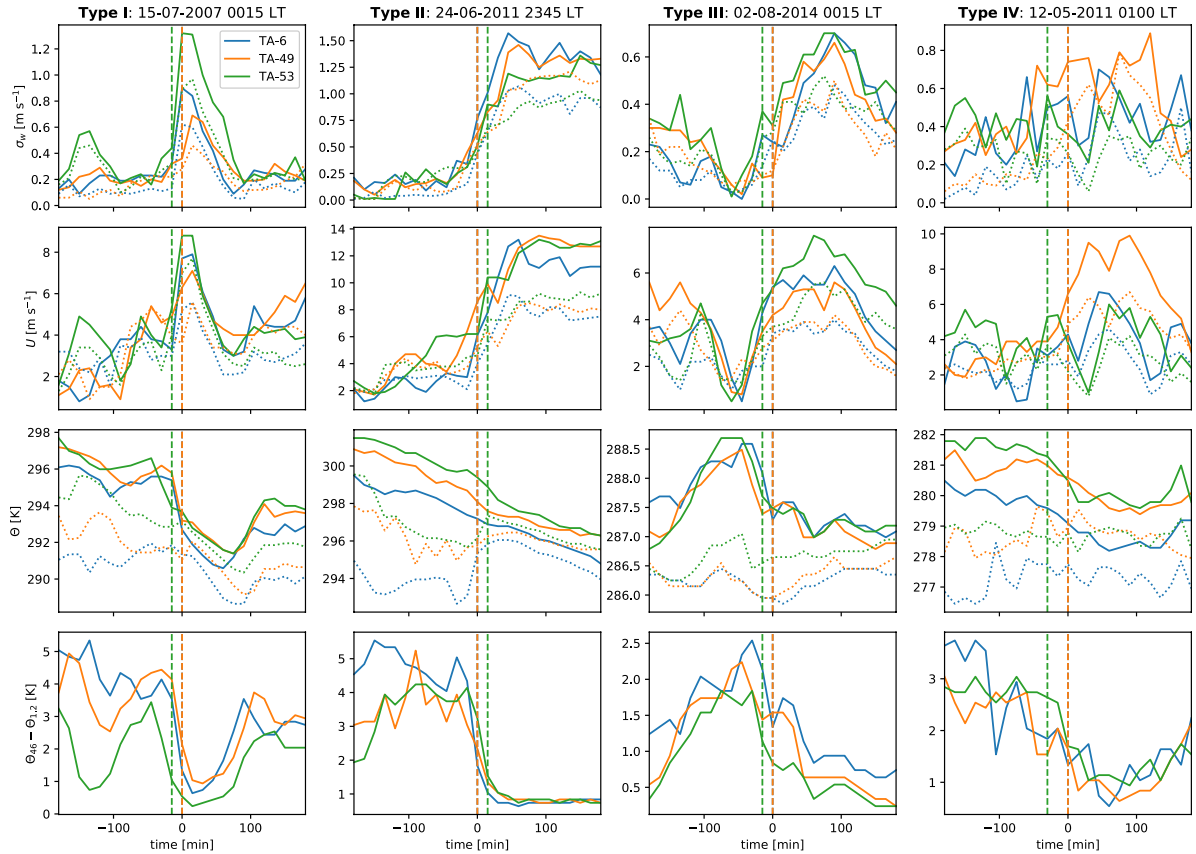

Fig. 11 Four different types of turbulence recovery events occurring contemporaneously at all tower stations (columns;). The evolutions of the fluctuations of the vertical wind component ( $\sigma w$; first row), wind speeds $(U$, second row), potential temperatures $(\Theta$; third row), and stratification $(\Theta 46-\Theta 1.2$; fourth row) are depicted in the $3 \mathrm{~h}$ before and after the vSBL to wSBL transitions with respect to the timing of the transitions at TA-6 (time zero referring to the different dates; LT $=$ UTC $-6 \mathrm{~h}$ ). The times of the vSBL to wSBL transitions at TA-49 and TA-53 is indicated by vertical dashed lines. Solid and dotted lines illustrate measurements respectively at $46 \mathrm{~m}$ and closest to the surface (respectively $11.5 \mathrm{~m}$ and $1.2 \mathrm{~m}$ for measurements related to wind speeds and temperature). Note that the vertical axes across all plots differ

Publisher's Note Springer Nature remains neutral with regard to jurisdictional claims in published maps and institutional affiliations. 